S. Garg • J. Rosenstock • B. L. Silverman • B. Sun •

C. S. Konkoy $\cdot$ A. de la Peña $\cdot$ D. B. Muchmore

\title{
Efficacy and safety of preprandial human insulin inhalation powder versus injectable insulin in patients with type 1 diabetes
}

Received: 1 November 2005 / Accepted: 8 December 2005 / Published online: 28 February 2006

C) Springer-Verlag 2006

\begin{abstract}
Aims/hypothesis: The efficacy and safety of human insulin inhalation powder (HIIP) plus insulin glargine were compared to subcutaneously injected insulin (SC insulin) plus insulin glargine in patients with type 1 diabetes. Methods: This was a randomised, open-label crossover study in which one group of patients received preprandial HIIP plus insulin glargine for 12 weeks, followed by the same duration with preprandial SC insulin (lispro or regular) plus insulin glargine. Another group of patients received the reverse treatment sequence. The trial was designed as a non-inferiority comparison of the two treatments for effect on $\mathrm{HbA}_{1 \mathrm{c}}$; blood glucose levels were also monitored. Safety assessments included adverse event reporting and hypoglycaemic events. Results: $\mathrm{HbA}_{1 \mathrm{c}}$ at endpoint was $7.95 \pm 0.12 \%$ for the HIIP treatment and $8.06 \pm 0.12 \%$ for the SC insulin treatment; mean changes from baseline to endpoint were -0.08 and $0.00 \%$, respectively, $(p=\mathrm{NS})$. The upper limit of the $95 \%$ CI of mean difference in $\mathrm{HbA}_{1 \mathrm{c}}$ between the two treatments was $0.02 \%$, indicating that HIIP was not inferior relative to SC insulin, as measured against the pre-defined margin of $0.3 \%$. Fasting blood glucose was significantly lower for HIIP treatment $(8.09 \pm 0.33 \mathrm{mmol} / \mathrm{l} ; n=117)$ than for SC insulin treatment $(9.05 \pm 0.33 \mathrm{mmol} / \mathrm{l} ; n=111)(p=0.01)$. Safety profiles were comparable between the two treat-
\end{abstract}

\author{
S. Garg $(\bowtie)$ \\ Department of Medicine and Paediatrics, Barbara Davis Center \\ for Diabetes at the University of Colorado Health Sciences \\ Center, Mail stop A140, \\ P.O. Box 6511 Aurora, CO 80045, USA \\ e-mail: satish.garg@uchsc.edu \\ Tel.: +1-303-7246713 \\ Fax: +1-303-7246784 \\ J. Rosenstock \\ Dallas Diabetes and Endocrine Center, Dallas, TX, USA
}

B. L. Silverman

Alkermes, Cambridge, MA, USA

B. Sun · C. S. Konkoy · A. de la Peña · D. B. Muchmore Lilly Research Laboratories, Lilly Corporate Center,

Indianapolis, IN, USA ments. The rate of any hypoglycaemia (least-squares mean adjusted for 30 days \pm SE) was $8.9 \pm 0.7$ and $8.2 \pm 0.8$ for HIIP and SC insulin treatments, respectively, $(p=0.29)$. The rate of nocturnal hypoglycaemia was greater for HIIP (4.2 \pm 0.4$)$ than for SC insulin $(2.7 \pm 0.4 ; p<0.001)$ Conclusions/interpretation: HIIP was similar in efficacy to SC insulin for glycaemic control in type 1 diabetes mellitus. The two treatments had comparable safety profiles.

Keywords Drug delivery $\cdot \mathrm{HbA}_{1 \mathrm{c}} \cdot$ Hyperglycaemia Inhaled insulin · Injection · Pulmonary .

Type 1 diabetes mellitus

Abbreviations $\mathrm{DL}_{\mathrm{CO}}$ : diffusing capacity for carbon monoxide $\cdot$ FBG: fasting blood glucose $\cdot$ FEV1: forced expiratory volume in $1 \mathrm{~s} \cdot \mathrm{FPG}$ : fasting plasma glucose HIIP: human insulin inhalation powder - LSM: leastsquares mean - SC: subcutaneously injected - SMBG: selfmonitoring blood glucose $\cdot$ TLV: total lung volume

\section{Introduction}

Insulin therapy in patients with diabetes often involves multiple daily injections of insulin, which, from the patient perspective, has several disadvantages including physical discomfort and inconvenience, as well as technical issues regarding drug administration $[1,2]$. Many patients dislike such injection regimens [3], possibly resulting in poor compliance and suboptimal glycaemic control [4]. Inhaled insulin represents a promising and practical alternative way of insulin delivery [5], with demonstrated proof of principle for effective pulmonary delivery in clinical trials involving patients with diabetes [4]. Multiple studies have shown that such patients prefer inhaled to injected regimens $[1,6-8]$, which may lead to greater patient acceptance of daily insulin administration and improved therapeutic outcomes.

Spray-drying technology has enabled production of large, low-density particles suitable for the delivery of 
therapeutic doses of human recombinant insulin via the pulmonary route. The combination of large particle size and low density has several advantages for the development of an aerosol with optimal properties for systemic absorption. These properties include: (1) reduced tendency to aggregate relative to smaller particles; (2) increased deposition into alveoli; and (3) reduced capacity for phagocytic clearance once deposition into the deep lung has occurred, leading to transport of insulin across the pulmonary capillary endothelium [9]. The high dispersibility of such an aerosol enables delivery of a wide range of doses using a simple dry-powder inhaler (Fig. 1). In addition to optimisation of particle attributes, an ideal aerosol delivery system should include an inhaler that is small, breath-actuated, and easy to use.

A previous study has shown that the human inhaled insulin powder (HIIP) used in the present study is well tolerated and has a good safety profile [10]. Moreover, HIIP demonstrated a broad range of dose proportionality relative to subcutaneously injected insulin lispro, as well as comparable glucodynamic effects and intra-subject variability. Here we report safety and efficacy findings of HIIP compared to subcutaneously injected (SC) insulin in patients with type 1 diabetes. This study represents the first investigation of efficacy with a small, breath-actuated delivery device using AIR particle technology $[9,11,12]$ (Alkermes, Cambridge, MA, USA). The study was designed with moderate-intensity treatment goals to ensure patient safety and a crossover design to determine treatment preference [13], which will be reported separately.

\section{Subjects and methods}

Patients

A total of 137 patients with type 1 diabetes of at least 24 months duration were enrolled. Diagnosis was confirmed by C-peptide concentration of $<165 \mathrm{pmol} / \mathrm{l}$. At screening, per eligibility criteria, $\mathrm{HbA}_{1 \mathrm{c}}$ values were $\geq 7$

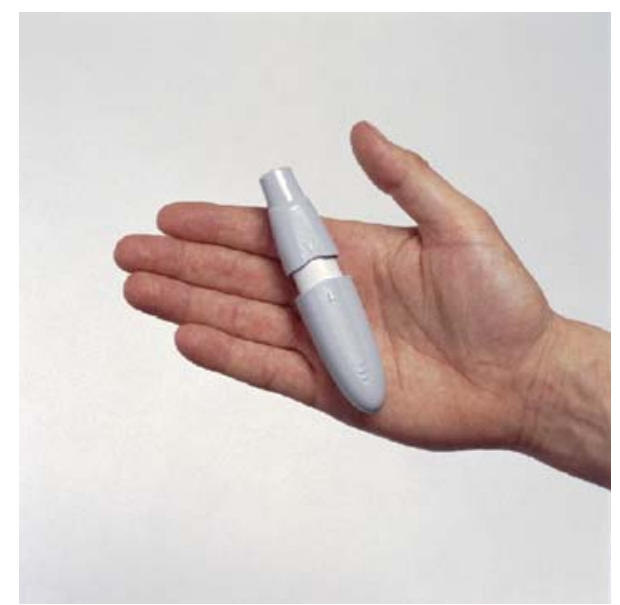

Fig. 1 The Lilly/Alkermes inhaled insulin delivery system used in the present study and $\leq 12 \%$ and patients had been currently injecting regular human insulin or insulin lispro for at least 4 weeks prior to study entry. Patients had pulmonary function tests within the normal range, i.e. forced expiratory volume in $1 \mathrm{~s}$ $\left(\mathrm{FEV}_{1}\right)$, forced vital capacity, and diffusing capacity for carbon monoxide $\left(\mathrm{DL}_{\mathrm{CO}}\right) \geq 75$ and $\leq 125 \%$ of predicted values. Patients were excluded from participation for the following reasons: history of asthma, atopy, or allergic rhinitis; upper respiratory infection within 4 weeks prior to screening; lower respiratory infection within 5 months prior to screening; chronic cough; clinical signs or symptoms of liver disease; renal dialysis, history of renal transplantation, or serum creatinine $\geq 0.13 \mathrm{mmol} / 1$ (males) or $\geq 0.12 \mathrm{mmol} / 1$ (females); history of angina, myocardial infarction, or New York Heart Association Class III/IV cardiac disease within 2 years prior to study entry; congestive heart failure requiring pharmacotherapy; advanced autonomic neuropathy; history of severe hypoglycaemia; confirmed gastroparesis; treatment for malignancy, excluding basal or squamous cell carcinoma; or systemic glucocorticoid therapy (except topical preparations). Participants had to be nonsmokers for at least 1 year, with a negative serum cotinine at screening. This phase II trial was conducted in agreement with the Declaration of Helsinki and the International Council on Harmonisation Guidelines to Good Clinical Practice. Patients signed informed consent prior to participation in the study.

\section{Study design}

The study was an open-label, 2-period crossover study conducted at 24 centres in the USA. During the 1-week lead-in period patients were instructed to inject regular human insulin or insulin lispro, as used prior to study entry, before meals, and insulin glargine at bedtime. Patients were randomised to one of two treatment sequences. One group received 12 weeks of preprandial HIIP plus insulin glargine daily, followed by 12 weeks of preprandial injectable insulin (SC insulin, i.e. regular human insulin or insulin lispro) plus insulin glargine daily. The other group received the same treatments in reverse order.

\section{Treatments}

\section{HIIP}

HIIP (of recombinant DNA origin) was formulated as a dry powder (AIR particle technology; Alkermes, Cambridge, MA, USA). Capsules were available in 2 dose strengths, low and medium. The low-dose capsules were filled with $0.9 \mathrm{mg}$ of insulin, equivalent to $2 \mathrm{U}$ of SC insulin. Mediumdose capsules were filled with $2.6 \mathrm{mg}$ of insulin, equivalent to $6 \mathrm{U}$ of SC insulin [10]. Capsules were loaded into a breath-actuated inhaler (pre-commercial model), which contained a puncturing mechanism to allow release and dispersal of insulin from the capsule without requiring an external energy source. Patients requiring preprandial 
insulin doses above $6 \mathrm{U}$ performed multiple inhalations to achieve the required dosage.

\section{Administration and dose adjustments}

Patients were instructed to follow these guidelines for timing of insulin administration: regular human insulin, 30-60 min before meals; insulin lispro 0 to $15 \mathrm{~min}$ before meals; HIIP 0 to 15 min before meals. Insulin glargine (subcutaneous) was administered at bedtime.

Dose adjustments were performed to achieve the following blood glucose concentration goals:

- Fasting and preprandial $\leq 7.8 \mathrm{mmol} / \mathrm{l}(140 \mathrm{mg} / \mathrm{dl})$

- 2-h postprandial $\leq 8.9 \mathrm{mmol} / \mathrm{l}(160 \mathrm{mg} / \mathrm{dl})$

Insulin dose (both preprandial and insulin glargine) was dependent upon the individual requirements of the patient and could be adjusted at any time during the study.

\section{Efficacy and safety measurements}

A central laboratory was used to analyse laboratory samples. $\mathrm{HbA}_{1 \mathrm{c}}$ levels were measured by high performance liquid chromatography (Bio-Rad Variant II; BioRad, Hercules, CA, USA), as certified by the National Glycohaemoglobin Standardisation Program [14]. Sevenpoint self-monitoring of blood glucose (SMBG) profiles were performed by the patient using a blood glucose meter (Accu-Chek Compact; Roche Diagnostics, Indianapolis, IN, USA) provided for the study. Patients were instructed to perform the test before breakfast, lunch, and evening meals; $2 \mathrm{~h}$ after breakfast, lunch, and evening meals; and at bedtime before injecting insulin glargine. The values and times of SMBG were recorded in patient diaries; insulin doses and times of administration, episodes of hypoglycaemia, illnesses, and concomitant medications were also recorded.

Safety assessments consisted of adverse event reporting; laboratory abnormalities; vital signs and body weight; hypoglycaemic episodes; and tests of pulmonary function. Adverse events were coded with a MedDRA dictionary. Hypoglycaemic episodes were defined by either (1) symptoms of hypoglycaemia as reported by the patient, or (2) SMBG of $<3.5 \mathrm{mmol} / 1(63 \mathrm{mg} / \mathrm{dl})$, regardless of the presence of hypoglycaemic signs or symptoms. Nocturnal hypoglycaemia was defined as any hypoglycaemic event that occurred between bedtime, after insulin glargine administration, and the following morning before administration of the prandial insulin. Severe hypoglycaemia was defined as an episode in which the patient required the assistance of another person and which was associated with either a blood glucose level $<2.8 \mathrm{mmol} / 1(50 \mathrm{mg} / \mathrm{dl})$ or prompt recovery after oral carbohydrate, glucagon or intravenous glucose. Data regarding hypoglycaemic episodes were drawn from patient diaries, in which patients were instructed to record symptoms, SMBG levels, and treatment.
Pulmonary function tests included FEV1, forced vital capacity, total lung volume (TLV) (performed only at select study sites), and $\mathrm{DL}_{\mathrm{CO}}$. Pulmonary function tests were assessed using guidelines established by the American Thoracic Society [15]. DL $\mathrm{CO}_{\mathrm{CO}}$ was adjusted for haemoglobin concentration via the following equations:

Males : $\mathrm{DL}_{\mathrm{CO} \text { corrected }}=\mathrm{DL}_{\mathrm{CO} \text { uncorrected }}(10.22+\mathrm{Hb}) / 1.7 \mathrm{Hb}$ Females : $\mathrm{DL}_{\mathrm{CO} \text { corrected }}=\mathrm{DL}_{\mathrm{CO} \text { uncorrected }}(9.38+\mathrm{Hb}) / 1.7 \mathrm{Hb}$

Pulmonary function tests were reviewed by a central quality-assurance monitor and assessed for compliance with American Thoracic Society testing standards [15].

Data analysis

The primary objective of this study was to test noninferiority of HIIP compared to SC insulin treatment. The analysis was based on an intent-to-treat sample, which included all randomised patients. The primary efficacy variable was $\mathrm{HbA}_{1 \mathrm{c}}$ at the end of each treatment period. Noninferiority of HIIP to SC insulin treatment was demonstrated if the upper limit of the one-sided 95\% CI of treatment difference was less than $0.3 \%$ as defined in the protocol. The CI was derived from ANOVA model for crossover study design. For other continuous efficacy and safety variables, a similar ANOVA model was performed to compare treatment differences. Fisher's exact test was used for crossover analyses of categorical variables. For missing post-baseline measurements, endpoint was defined by the last observation carried forward within each period. No carry-over effects were observed for the analyses described here.

\section{Results}

Patient characteristics and disposition

Of the 137 patients enrolled in the study, 68 patients first received HIIP, then crossed over to SC insulin, whereas 69 received the same treatments in reverse order. The majority of patients were women $(n=73 ; 53.3 \%)$ and of white European origin $(n=120 ; 87.6 \%)$. Mean age was $39.0 \pm 12.2$ years. Mean BMI was $28.1 \pm 4.8 \mathrm{~kg} / \mathrm{m}^{2}$. Thirty patients $(21.9 \%)$ were former smokers. Baseline $\mathrm{HbA}_{1 \mathrm{c}}$ $($ mean \pm SD) was $8.1 \pm 1.1 \%(n=137)$. None of the above patient characteristics at baseline differed significantly with respect to treatment sequence. Approximately $70 \%$ $(n=94)$ of patients were using insulin lispro and $30 \%$ $(n=43)$ were using regular human insulin; baseline $\mathrm{HbA}_{1 \mathrm{c}}$ values for these patient groups were $8.1 \pm 1.0$ and $8.0 \pm 1.3 \%$, respectively. Patient disposition is diagrammed in Fig. 2. A total of 119 patients $(87 \%)$ completed both treatment phases of the study; the percentage of completers did not differ significantly between treatment sequences. 


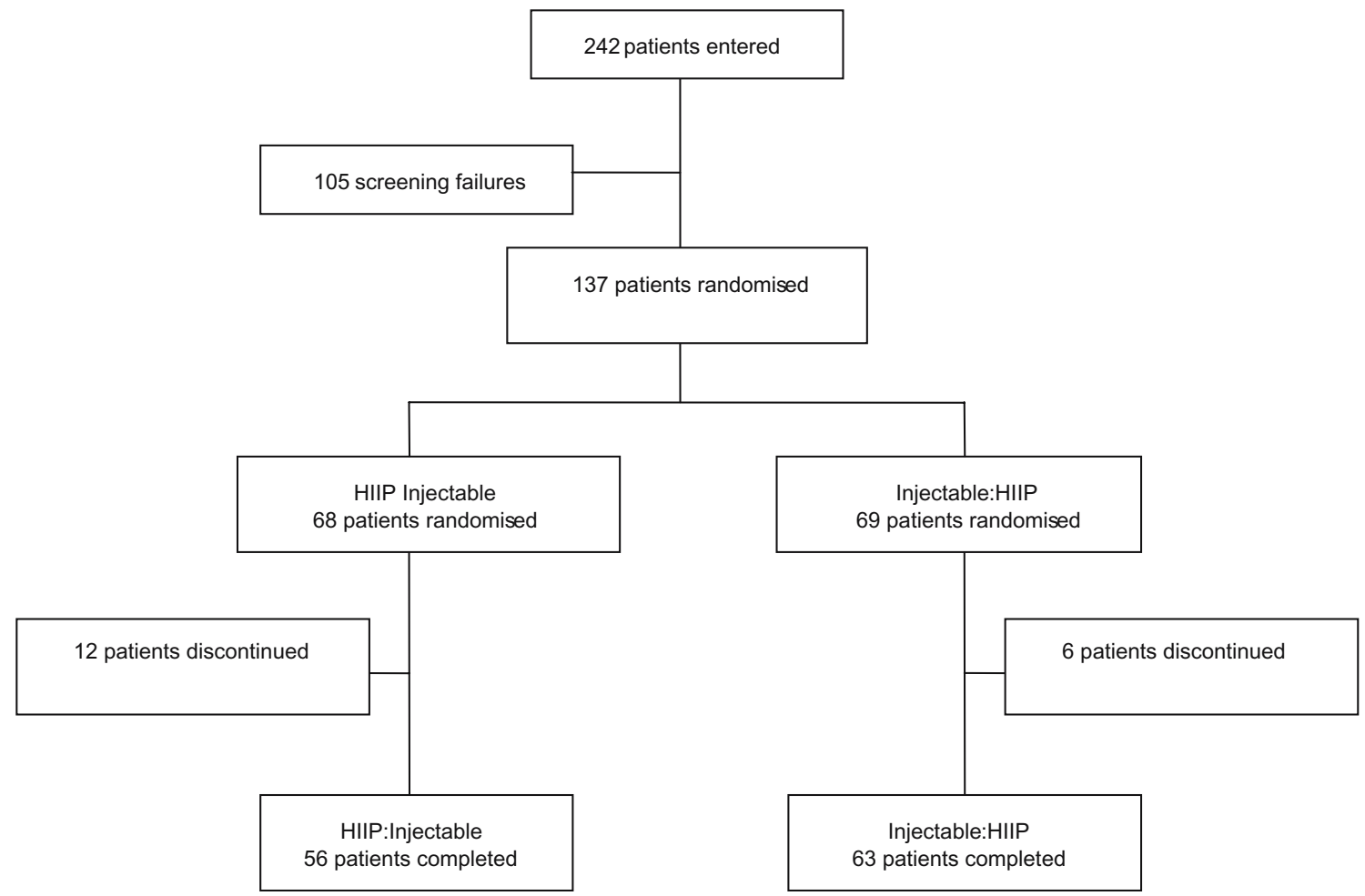

Fig. 2 Flowchart of study procedure

Glycaemic control

$H b A_{l c}$

For combined treatment periods, $\mathrm{HbA}_{1 \mathrm{c}}$ values (leastsquares mean $[\mathrm{LSM}] \pm \mathrm{SE}$ ) at endpoint were similar for the HIIP group $(7.95 \pm 0.12 \% ; n=127)$ and the $\mathrm{SC}$ insulin group $(8.06 \pm 0.12 \% ; n=119)$ (Fig. 3). The upper limit of the onesided $95 \%$ CI was 0.02 (LSM treatment difference [HIIP$\mathrm{SC}]=-0.10 ; p=0.17$ ). Changes from baseline (means $\pm \mathrm{SD}$ ) were similar between the treatment groups $(-0.08 \pm 0.80$ and $0.00 \pm 0.72 \%$, respectively). Further analysis showed $\mathrm{HbA}_{1 \mathrm{c}}$ at endpoint was not significantly different between the HIIP and SC insulin groups within each treatment period $(p=0.74$ and $p=0.39$ for periods 1 and 2, respectively). Furthermore, a similar proportion of patients for each group achieved the $\mathrm{HbA}_{1 \mathrm{c}}$ target $(\leq 7 \%)$ at endpoint (26/127 [20.5\%] and 26/119 [21.8\%] for HIIP and SC insulin groups, respectively).

Subgroup analyses were conducted to examine possible effects of type of SC insulin on treatment outcome. For patients whose SC insulin was regular human insulin, the $\mathrm{LSM} \pm \mathrm{SE}$ at endpoint was $8.34 \pm 0.21$ and $8.32 \pm 0.21 \%$ for the HIIP $(n=40)$ and SC insulin groups $(n=37)$, respectively, ( $p=0.90$; upper limit of $95 \%$ CI 0.24 ) (Fig. 3), with similar changes from baseline (means $\pm \mathrm{SD}$ : $0.08 \pm 0.78$ and $0.00 \pm 0.79 \%$, respectively). For patients whose SC insulin was insulin lispro, the $\mathrm{LSM} \pm \mathrm{SE}$ at endpoint was $7.89 \pm 0.16$ and $8.05 \pm 0.16 \%$ for the HIIP $(n=87)$ and SC insulin $(n=82)$ groups, respectively, ( $p=0.08$; upper limit of $95 \%$ CI $-0.01)$, with similar changes from baseline $(0.16 \pm 0.80$ and $0.00 \pm 0.69 \%$, respectively).

\section{Blood glucose}

Based on SMBG reporting in the patient diaries (Fig. 4), fasting blood glucose (FBG, morning preprandial measurement; $\mathrm{LSM} \pm \mathrm{SE}$ ) was significantly lower for the HIIP treatment $(8.09 \pm 0.33 \mathrm{mmol} / \mathrm{l} ; n=117)$ than for the $\mathrm{SC}$

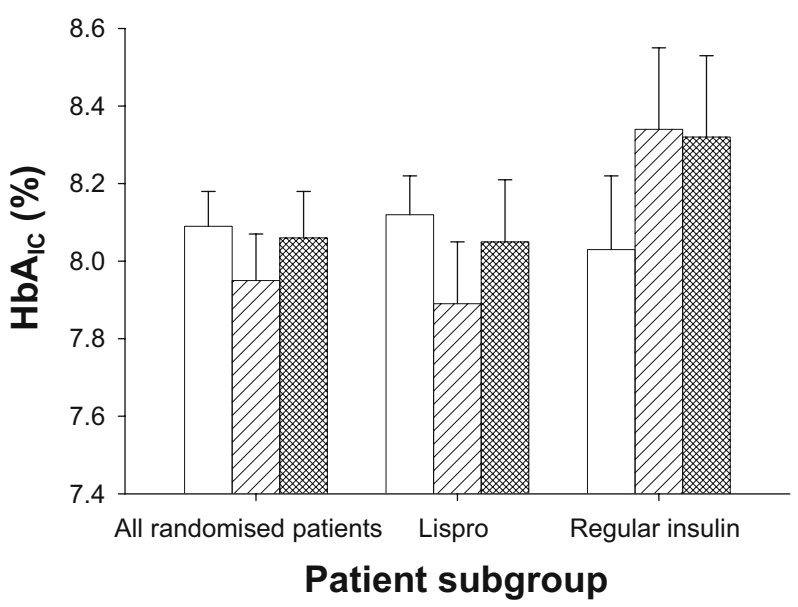

Fig. $3 \mathrm{HbA}_{1 \mathrm{c}}$ at baseline and endpoint for all randomised patients and for SC insulin subgroups. Patients were randomised $(n=137)$ to receive either HIIP ( $n=127$ at endpoint) or SC insulin $(n=119$ at endpoint) for 12 weeks, then crossed over to receive the other treatment for 12 weeks. During the SC insulin phase, patients received either insulin lispro ( $n=94$ baseline; $n=87$ endpoint HIIP; $n=82$ endpoint SC) or regular human insulin ( $n=43$ baseline; $n=40$ endpoint HIIP; $n=37$ endpoint SC) according to their pre-treatment regimen. Results are least squares means $\pm \mathrm{SE}$, except for baseline (means $\pm \mathrm{SE}$ ). White bars, baseline; single crosshatch, endpoint HIIP; double crosshatch, endpoint SC insulin 


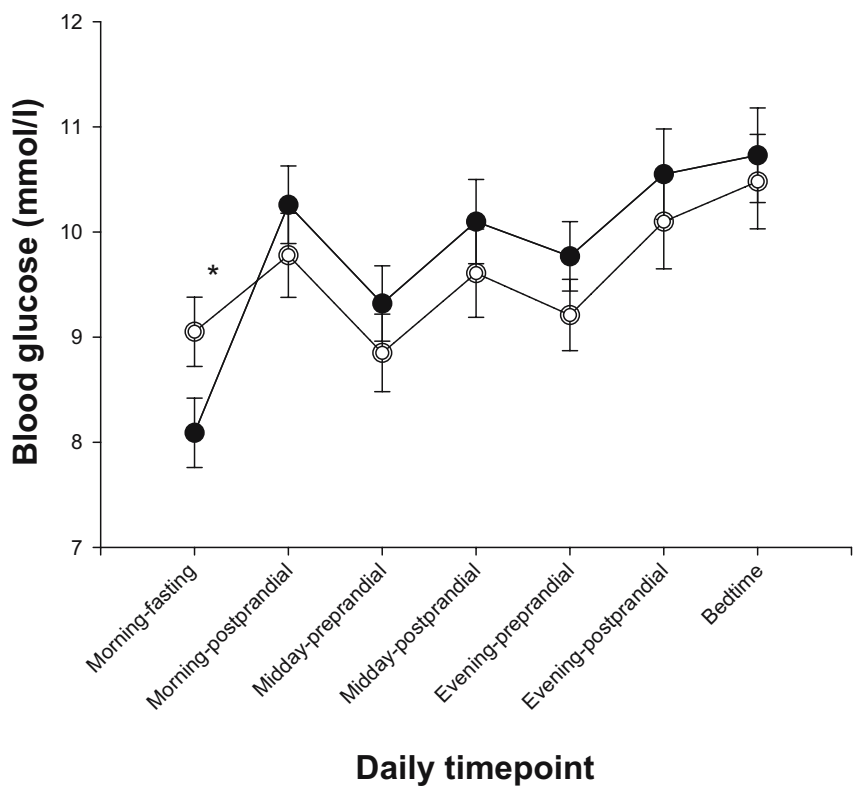

Fig. 4 Daily blood glucose measurements. Patients were randomised to receive either HIIP or SC insulin for 12 weeks, then crossed over to receive the other treatment for 12 weeks. Values are sevenpoint self-monitored blood glucose concentrations. Results are least squares means \pm SE. Solid circles, HIIP; open circles, SC insulin. $* p=0.01$ vs SC insulin

insulin treatment $(9.05 \pm 0.33 \mathrm{mmol} / 1 ; p=0.01 ; n=111)$. Blood glucose levels showed an upward trend over the course of the daily measurement with bedtime levels of $10.73 \pm 0.45 \mathrm{mmol} / \mathrm{l}$ for the HIIP treatment $(n=113)$ and $10.48 \pm 0.47 \mathrm{mmol} / \mathrm{l}$ for the SC insulin treatment $(n=106)$. Although average daily values for the 2 -h excursion blood glucose levels appeared to be lower for SC insulin treatment $(0.71 \pm 0.32 \mathrm{mmol} / \mathrm{l} ; n=99)$ than for HIIP treatment $(1.31 \pm 0.30 \mathrm{mmol} / 1 ; n=109)$, the difference was not statistically significant $(p=0.08)$. Morning 2-h glucose excursion blood glucose levels were significantly higher for HIIP treatment $(2.24 \pm 0.46 \mathrm{mmol} / \mathrm{l} ; n=106)$ than for SC insulin treatment $(0.97 \pm 0.5 \mathrm{mmol} / \mathrm{l} ; p=0.04 ; n=96)$. There were no statistically significant differences between treatments for the midday or evening preprandial, postprandial, or bedtime blood glucose levels.
Daily insulin requirements

Total daily treatment doses for rapid-acting insulin, daily insulin glargine doses, and total daily doses for HIIP and SC insulin treatments are shown in Table 1. Daily requirements for insulin at the end of each period did not differ significantly between treatments for any of the mealtime doses or for bedtime (glargine) doses.

\section{Safety}

The incidence of treatment-emergent adverse events did not differ significantly between HIIP $(79 / 133 ; 59.4 \%)$ and SC insulin treatments $(79 / 126 ; 62.7 \%)(p=0.99)$. Cough was the most frequently reported adverse event during HIIP treatment and was the only adverse event whose incidence differed significantly between treatments (13.5 and $4.0 \%$ of patients during the HIIP and SC insulin treatments, respectively; $p=0.005$ ). Three serious treatment-emergent adverse events were reported during treatment with SC insulin, one of which was due to hypoglycaemia. Two patients discontinued the study due to adverse events: one patient discontinued during the HIIP phase because of hypoglycaemia and another patient discontinued during the SC insulin phase because of viral encephalitis. Only small changes in weight were observed from baseline to endpoint for the two treatments $(0.18 \mathrm{~kg}$ for both HIIP and SC insulin).

\section{Hypoglycaemia}

The HIIP and SC insulin treatments showed similar rates of hypoglycaemia for entire treatment periods (LSM $\pm \mathrm{SE}$ : $8.9 \pm 0.7$ and $8.2 \pm 0.8$ events per 30 days, respectively; $p=0.29$ ). Significant between-treatment differences for any hypoglycaemia were reported only at early timepoints in the study (Fig. 5a), with higher rates of hypoglycaemia during HIIP than during SC insulin treatment at Week 2 $(13.1 \pm 1.0$ vs $9.0 \pm 1.0$ events per 30 days, respectively; $p<0.001)$ and at Week $4(11.1 \pm 0.9$ vs $8.8 \pm 0.9$ events per 30 days, respectively; $p=0.004)$. The incidence of severe

Table 1 Insulin dose requirements

\begin{tabular}{|c|c|c|c|c|}
\hline & HIIP $[n=116]$ & & $\mathrm{SC}$ in & \\
\hline \multicolumn{5}{|l|}{ Bolus insulin dose } \\
\hline Breakfast & 7.5 & $(0.6)$ & 7.3 & $(0.6)$ \\
\hline Dinner & 8.6 & $(0.6)$ & 9.1 & $(0.6)$ \\
\hline Bedtime supplement & 1.0 & $(0.2)$ & 1.1 & $(0.2)$ \\
\hline
\end{tabular}

Data expressed in insulin units, least squares means $(S E)$. No significant differences were observed between the treatments for any of the dosing parameters listed here

HIIP doses represent SC unit equivalents that were determined previously in a euglycaemic clamp study conducted in normal volunteers [10] 
hypoglycaemia was similar for the HIIP and SC insulin treatment periods (12 patients [9.0\%] and 11 patients [8.7\%], respectively; $p=0.999)$, with similar rates for entire treatment $(0.05 \pm 0.03$ [both treatments] per 30 days or $60 \pm 36$ per 100 patient years [both treatments]; $p=0.829$ ).

The rate of nocturnal hypoglycaemia was significantly higher during HIIP treatment than during SC insulin treatment (LSM \pm SE: $4.2 \pm 0.4$ vs. $2.7 \pm 0.4$ events per 30 days), both overall and at each timepoint (Fig. 5b). Differences between the two treatments remained statistically significant at all timepoints in the insulin lispro subgroup (Fig. 5c), but were not statistically significant for any of the timepoints in the regular insulin group (Fig. 5d).
Insulin antibodies

At endpoint, insulin-specific antibody titre ( $\mathrm{LSM} \pm \mathrm{SE}$ ) was $1.7 \pm 0.4 \%$ for the HIIP treatment period and $0.6 \pm 0.4 \%$ for the SC insulin treatment period $(p<0.001$ between treatments), with mean changes from baseline of 1.3 and $0.03 \%$, respectively.

Pulmonary function

The results of pulmonary function tests are shown in Table 2. No statistically significant treatment differences a

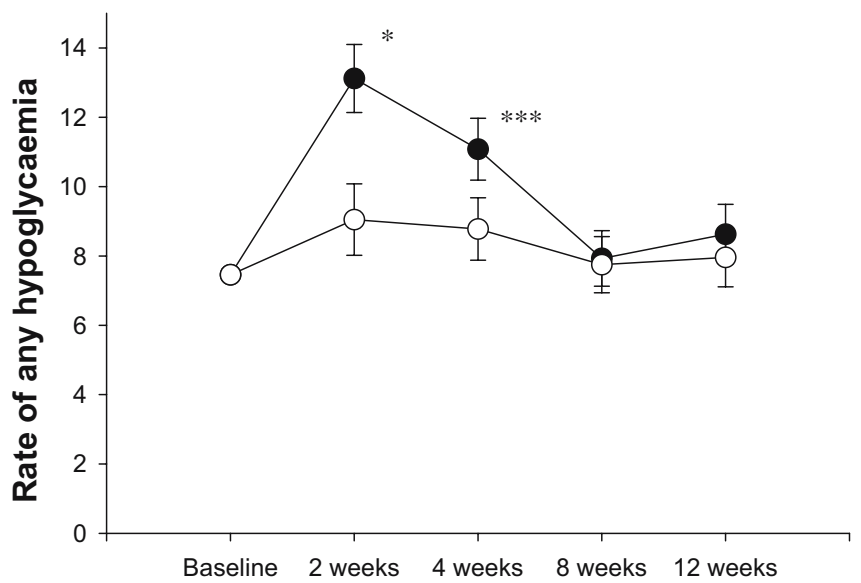

Duration of treatment

C

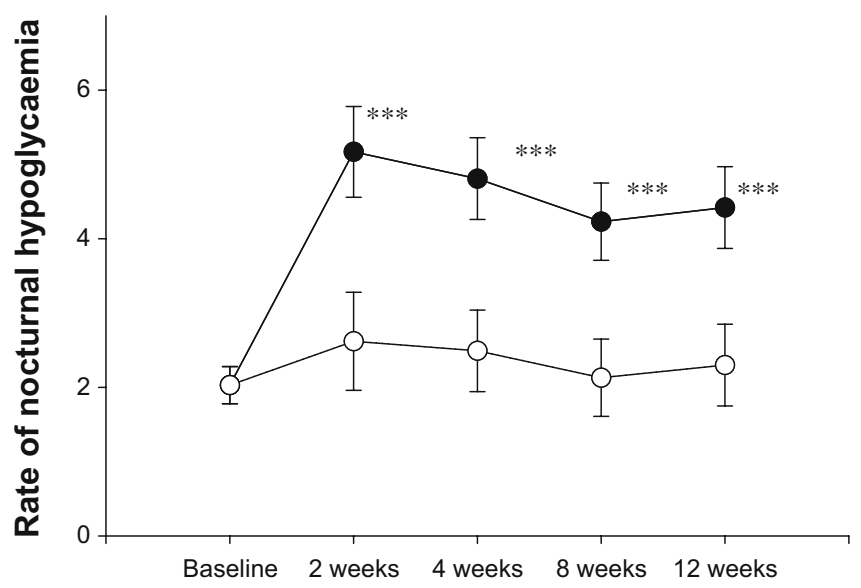

Duration of treatment

Fig. 5 Rate of any hypoglycaemia (a) and nocturnal hypoglycaemic episodes (b-d) over time. Rates are expressed as least squares means $\pm \mathrm{SE}$, except for baseline (means $\pm \mathrm{SE}$ ), and were adjusted to a 30 -day value. (a) Any hypoglycaemia, ${ }_{p}<0.005$, $* * * p<0.001$; b

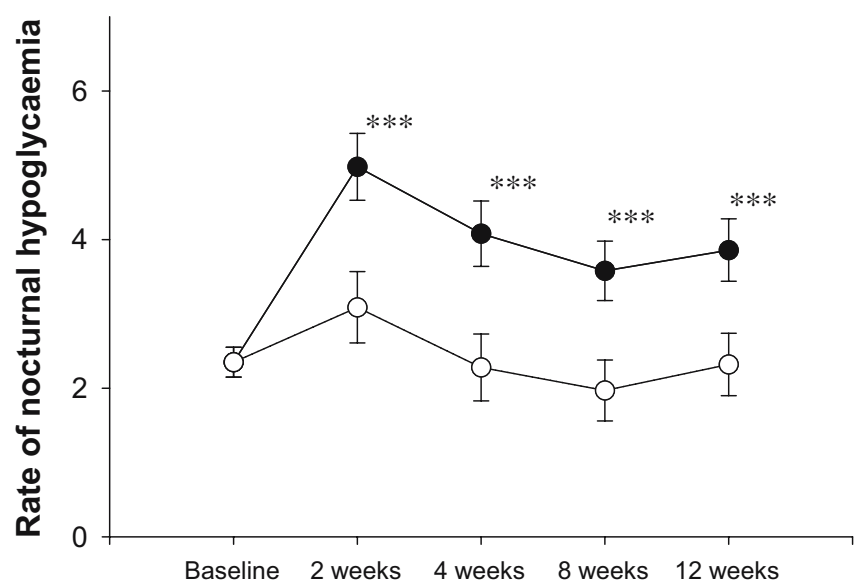

Duration of Treatment

d

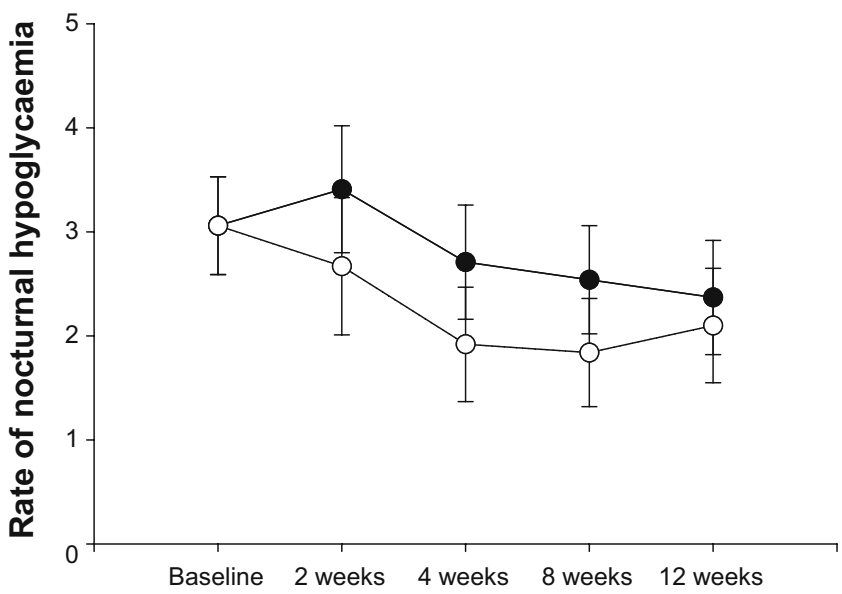

Duration of treatment

(b) Nocturnal hypoglycaemia, all randomised patients, $* * * p<0.001$; (c) Nocturnal hypoglycaemia, patients receiving insulin lispro, $* * * p<0.001$; (d) Nocturnal hypoglycaemia, patients receiving regular human insulin. Solid circles, HIIP; open circles, SC insulin 
were observed between HIIP and SC insulin for $\mathrm{FEV}_{1}$, forced vital capacity, or TLV. $\mathrm{DL}_{\mathrm{CO}}$ significantly decreased from baseline with HIIP treatment compared with SC insulin treatment $(p<0.001)$. Mean changes from baseline $\left(-1.45\right.$ and $-0.44 \mathrm{ml} \mathrm{min}^{-1} \mathrm{mmHg}^{-1}$ for HIIP and SC insulin, respectively) were, however, both small and not considered clinically significant. Moreover, the reduction in $\mathrm{DL}_{\mathrm{CO}}$ measured after treatment with HIIP returned toward baseline when these patients were crossed over to SC insulin $(n=68)(27.1 \pm 6.9,25.8 \pm 6.9$, and $27.1 \pm 7.7 \mathrm{ml}$ $\min ^{-1} \mathrm{mmHg}^{-1}$ at baseline, endpoint of HIIP treatment, and endpoint of SC insulin treatment, respectively), indicating that this change was reversible.

\section{Discussion}

This was the first study to compare the use of inhaled insulin with that of subcutaneous insulin in type 1 diabetic patients on either insulin lispro or regular human insulin at mealtimes and insulin glargine for basal coverage. Our primary finding was that inhaled insulin+insulin glargine was not inferior to standard treatment with subcutaneous insulin+insulin glargine with respect to endpoint $\mathrm{HbA}_{1 \mathrm{c}}$. Similar results were obtained, i.e. equivalent glycaemic control with respect to $\mathrm{HbA}_{1 \mathrm{c}}$, for subgroup analyses of patients on insulin lispro or regular human insulin. These results are consistent with those reported previously in randomised, parallel-group studies of 12-weeks [16] and 24-weeks duration [7, 17], in which reductions in $\mathrm{HbA}_{1 \mathrm{c}}$ were comparable for groups treated with mealtime inhaled insulin or subcutaneously injected regular human insulin. The results of these two previous studies [7, 17], however, are difficult to interpret since different basal insulins were used in the two treatment groups.

FBG values were significantly lower during HIIP treatment than during the SC insulin treatment, a finding which is similar to those of previous trials of a dry-powder insulin formulation, in which FPG was significantly lower after 24 weeks of treatment in the inhaled insulin group than in the SC insulin group [7, 17]. Lower FBG was not attributable to mean differences in insulin or glargine dosage. Although morning 2-h excursion blood glucose levels were significantly higher during HIIP treatment than during SC insulin treatment (due to significantly lower FBG before breakfast), all postprandial daily blood glucose values were similar between treatments. Furthermore, average daily values for the 2 -h excursion blood glucose levels were not significantly different between treatments. Together with the comparability of insulin dosing requirements between the two treatments, these results suggest that HIIP provided equivalent glycaemic control to that of $\mathrm{SC}$ insulin.

HIIP showed a satisfactory safety and tolerability profile, consistent with previous investigations of inhaled insulin in healthy volunteers [18-20], patients with type 1 diabetes [7, 16, 17] and type 2 diabetes [18, 21-23]. Cough, the most common respiratory side effect reported in previous trials of inhaled insulin [5], was the most frequently repeated adverse event related to treatment with HIIP. The event was judged to be mild in severity in the majority of cases (data not shown).

The rate of any hypoglycaemia was significantly higher for the HIIP group than for the SC insulin group after 2 and 4 weeks of treatment. Differences between the two treatments were transitory inasmuch as rates of hypoglycaemia were similar at endpoint. Endpoint values approached baseline values for both treatments (data not shown). These findings suggest that patients who are switched from SC insulin to HIIP learn over a few weeks of treatment to adjust their insulin dose, diet, and exercise so that the rate of hypoglycaemia returns to the baseline level. The rate of nocturnal hypoglycaemia was significantly higher in the HIIP group than in the SC insulin group throughout the study. Subgroup analyses revealed that rates of nocturnal hypoglycaemia appeared to depend on the type of mealtime insulin patients were using. Patients who were using insulin lispro had a significantly higher rate of

Table 2 Pulmonary function tests by treatment

\begin{tabular}{|c|c|c|c|c|c|}
\hline $\begin{array}{l}\text { Pulmonary function test } \\
\mathrm{FEV}_{1} \text { (1) }\end{array}$ & \multicolumn{2}{|c|}{ Baseline, mean (SD) } & \multicolumn{2}{|c|}{ Endpoint, mean (SD) } & $p$ value for treatment difference \\
\hline HIIP $(n=126)$ & 3.20 & $(0.84)$ & 3.13 & $(0.86)$ & 0.08 \\
\hline \multicolumn{6}{|l|}{ FVC (1) } \\
\hline HIIP $(n=126)$ & 3.97 & $(1.03)$ & 3.93 & $(1.06)$ & 0.76 \\
\hline SC insulin $(n=123)$ & 3.97 & $(1.03)$ & 3.94 & $(1.03)$ & \\
\hline HIIP $(n=125)$ & 27.0 & $(6.8)$ & 25.3 & $(6.7)$ & $<0.001$ \\
\hline SC insulin $(n=123)$ & 27.0 & $(6.8)$ & 26.6 & $(7.1)$ & \\
\hline \multicolumn{6}{|l|}{ TLC (l) } \\
\hline HIIP $(n=125)$ & 5.56 & $(1.35)$ & 5.44 & $(1.34)$ & 0.14 \\
\hline SC insulin $(n=123)$ & 5.56 & $(1.35)$ & 5.50 & $(1.18)$ & \\
\hline
\end{tabular}

Results are for combined periods. Number of patients is for patients for whom both baseline and endpoint values were available $\mathrm{FEV}_{1}$, forced expiratory volume in $1 \mathrm{~s}$; FVC, forced vital capacity, DL $\mathrm{CO}$, diffusing capacity of the lung for carbon monoxide; TLC, total lung capacity 
nocturnal hypoglycaemia during their HIIP treatment phase than during treatment with their usual SC-injectable insulin. In contrast, patients on regular human insulin had similar rates of nocturnal hypoglycaemia in both treatment phases. These results are consistent with the observation that serum insulin concentration returns more slowly to baseline for HIIP compared to SC lispro users (480 and 360 min, respectively) [10] and indicate that dosing regimens for HIIP may require minor adjustments to minimise the risk of nocturnal hypoglycaemia without sacrificing optimal control of FBG.

Insulin-specific antibodies increased over baseline values during the HIIP treatment period, a well-known finding from previous trials with various inhaled insulin formulations [24]. The increase in antibody binding observed here was not associated with changes in clinically meaningful variables. A recent study that pooled data from phase II and III trials showed that antibody formation from inhaled insulin (Exubera) was not associated with hypersensitivity reactions or with loss of glycaemic control or change in pulmonary function [25]. Together with another prospectively designed trial that assessed the impact of insulin antibody formation on glucodynamic variables and clinical safety in type 1 diabetic patients receiving inhaled insulin [26], none of these studies showed an association between increases in insulin antibody binding and adverse clinical sequelae.

Previous phase III trials in patients with type 1 diabetes showed small, nonprogressive decreases in $\mathrm{DL}_{\mathrm{CO}}$ among patients receiving of inhaled insulin [7, 17]. We observed a similar decrease in $\mathrm{DL}_{\mathrm{CO}}$ that appeared to be reversible for patients who first received HIIP, then crossed over to SC insulin. Although long-term evaluation of pulmonary function will be necessary to assess the overall safety profile of HIIP within the lung, preliminary results have shown that lung function is maintained in patients receiving a continuous regimen of inhaled insulin for up to 4 years [27].

In summary, results from this crossover study suggest that HIIP provided glycaemic control equivalent to that of SC insulin in patients with type 1 diabetes. HIIP demonstrated a safety profile comparable to that of SC insulin. Together with improved fasting glucose levels during the HIIP treatment period, the increased rate of nocturnal hypoglycaemia observed in this study for HIIP treatment relative to $\mathrm{SC}$ insulin treatment is consistent with previous reports showing that inhaled insulin formulations have a duration of activity comparable to that of regular insulin and longer than that of insulin lispro [10,20]. Patients with type 1 diabetes have expressed greater satisfaction with inhaled over injected insulin [1, 6, 7], suggesting that the availability of inhaled insulin may lead to better patient adherence to intensive insulin therapy. Additional studies are underway to investigate whether more intensive treatment with inhaled insulin can achieve the tight glycaemic control needed to delay or prevent diabetic complications.
Acknowledgements This work was sponsored by Eli Lilly and Company and is related to study protocol H7U-MC-IDAI. Parts of this work were presented at the 65th Annual Meeting and Scientific Sessions of the American Diabetes Association, San Diego, June 10 14, 2005 and at the 41st Annual Meeting of the European Association for the Study of Diabetes, Athens, Greece, September 12-15, 2005. S. Garg and J. Rosenstock are consultants/speakers to Eli Lilly and Company and have also received research grants from the company. B. L. Silverman is an employee and shareholder of Alkermes, Inc. B. Sun, C. S. Konkoy, A. de la Peña, and D. B. Muchmore are employees and shareholders of Eli Lilly and Company.

\section{References}

1. Gerber RA, Cappelleri JC, Kourides IA, Gelfand RA (2001) Treatment satisfaction with inhaled insulin in patients with type 1 diabetes: a randomized controlled trial. Diabetes Care 24:1556-1559

2. Hunt LM, Valenzuela MA, Pugh JA (1997) NIDDM patients' fears and hopes about insulin therapy: the basis of patient reluctance. Diabetes Care 20:292-298

3. Overmann H, Heinemann L (1999) Injection-meal interval: recommendations of diabetologists and how patients handle it. Diabetes Res Clin Pract 43:137-142

4. Barnett AH (2004) Exubera inhaled insulin: a review. Int J Clin Pract 58:394-401

5. Bindra S, Cefalu WT, Rosenstock J (2002) Inhaled insulin: a novel route for insulin delivery. Expert Opin Investig Drugs 11:687-691

6. Rosenstock J, Cappelleri JC, Bolinder B, Gerber RA (2004) Patient satisfaction and glycemic control after 1 year with inhaled insulin (Exubera) in patients with type 1 or type 2 diabetes. Diabetes Care 27:1318-1323

7. Quattrin T, Bélanger A, Bohannon NJV, Schwartz SL, for the Exubera Phase III Study Group (2004) Efficacy and safety of inhaled insulin (Exubera) compared with subcutaneous insulin therapy in patients with type 1 diabetes. Diabetes Care 27:2622-2627

8. Freemantle N, Blonde L, Duhot D et al (2005) Availability of inhaled insulin promotes greater perceived acceptance of insulin therapy in patients with type 2 diabetes. Diabetes Care 28:427-428

9. Edwards DA, Ben-Jebria A, Langer R (1998) Recent advances in pulmonary drug delivery using large, porous inhaled particles. J Appl Physiol 84:379-385

10. Rave K, Nosek L, de la Pena A et al (2005) Dose response of inhaled dry-powder insulin and dose equivalence to subcutaneous insulin lispro. Diabetes Care 28:2400-2405

11. Dunbar C, Scheuch G, Sommerer K, DeLong M, Verma A, Batycky R (2002) In vitro and in vivo dose delivery characteristics of large porous particles for inhalation. Int J Pharm 245:179-189

12. Valente A, Langer R, Stone HA, Edwards DA (2003) Recent advances in the development of an inhaled insulin product. Biodrugs 17:9-17

13. Ramadan NM (2002) Assessing the efficacy of drugs for the acute treatment of migraine. CNS Drugs 16:181-196

14. DCCT Research Group (1993) The effect of intensive treatment of diabetes on the development and progression of long-term complications of insulin-dependent diabetes mellitus. N Engl J Med 329:977-986

15. American Thoracic Society (1995) Standardization of spirometry, 1994, update. Am J Respir Crit Care Med 152:1107-1136

16. Skyler JS, Cefalu WT, Kourides IA et al, for the Inhaled Insulin Phase II Study Group (2001) Efficacy of inhaled human insulin in type 1 diabetes mellitus: a randomised proof-of-concept study. Lancet 357:331-335 
17. Skyler JS, Weinstock RS, Raskin P et al (2005) Use of inhaled insulin in a basal/bolus insulin regimen in type 1 diabetic subjects. Diabetes Care 28:1630-1635

18. Pfutzner A, Mann AE, Steiner SS (2002) Technosphere/Insulin - a new approach for effective delivery of human insulin via the pulmonary route. Diabetes Technol Ther 4:589-594

19. Rave K, Nosek L, Heinemann L, Gonzales C, Ernest CS, Muchmore DB (2004) Inhaled micronized crystalline human insulin using a dry powder inhaler: dose-response and timeaction profiles. Diabet Med 21:763-768

20. Rave K, Bott S, Heinemann L et al (2005) Time-action profile of inhaled insulin in comparison with subcutaneously injected insulin lispro and regular human insulin. Diabetes Care 28:1077-1082

21. Cefalu WT, Skyler JS, Kourides IA et al, Inhaled Insulin Study Group (2001) Inhaled human insulin treatment in patients with type 2 diabetes mellitus. Ann Intern Med 134:203-207

22. Hermansen K, Ronnemaa T, Petersen AH, Bellaire S, Adamson U (2004) Intensive therapy with inhaled insulin via the AERx insulin diabetes management system. Diabetes Care 27: $162-167$
23. Hollander PA, Blonde L, Rowe R et al, for the Exubera Phase III Study Group (2004) Efficacy and safety of inhaled insulin (Exubera) compared with subcutaneous insulin therapy in patients with type 2 diabetes. Diabetes Care 27:2356-2362

24. Heinemann L, Heise T (2004) Current status on the development of inhaled insulin. Br J Diabetes Vasc Dis 4:295-301

25. Fineberg SE, Kawabata T, Finco-Kent D, Liu C, Krasner A (2005) Antibody response to inhaled insulin in patients with type 1 or type 2 diabetes: an analysis of initial phase II and phase III inhaled insulin (Exubera) trials and a two-year extension trial. J Clin Endocrinol Metab 90:3287-3294

26. Heise T, Bott S, Tusek C et al (2005) The effect of insulin antibodies on the metabolic action of inhaled and subcutaneous insulin. Diabetes Care 28:2161-2169

27. Brain JD (2005) Unlocking the opportunity of tight glycaemic control. Inhaled insulin: safety. Diabetes Obes Metab 7 (Suppl 1):S14-S18 